\title{
A Study of Prognostic Markers for Dengue Infection
}

\author{
Ronak Parag Ajmera ${ }^{*}$, Sanjay V. Kulkarni' ${ }^{1}$ \\ ${ }^{1}$ Department of Medicine, M. S. Ramaiah Medical College, Bangalore, India
}

Corresponding Author: Ronak Parag Ajmera, MD, Department of Medicine, M. S. Ramaiah Medical College, Bangalore, India. Tel: +91-7021386921, Email: ronakajmera@hotmail.com, ronakajmera90@gmail.com

Received March 5, 2021; Accepted July 17, 2021; Online Published July 26, 2021

\begin{abstract}
Introduction: Early diagnosis and prognostication of infections such as dengue are crucial for better patient outcomes, as they help predict the likelihood of patients developing severe dengue, allowing more comprehensive patient triage and therapeutic interventions. This study aimed to determine clinical, laboratory, and radiological factors predicting prognosis in dengue infection.

Methods: This prospective observational study included 250 patients seropositive for dengue. They were classified into dengue fever (DF), dengue hemorrhagic fever (DHF), and dengue shock syndrome (DSS); and evaluated both on admission and at the end of their hospital course, the latter was performed for factors responsible for the progression of dengue to severe dengue. Data were statistically analyzed using R 3.6.1, with $P<0.05$ considered statistically significant.

Results: Final diagnosis correlated significantly with systolic blood pressure $(P=0.004)$, lowest platelet count $(P<0.001)$, serum glutamic-oxaloacetic transaminase $(P=0.001)$, urine protein $(P<0.001)$, urine red blood cells $(P<0.001)$, pleural effusion $(P=0.0064)$, serositis $(P<0.001)$, vomiting $(P<0.001)$, rash $(P<0.001)$, restlessness $(P<0.001)$, and bleeding manifestations $(P<0.001)$.

Conclusion: The prognosis of dengue is significantly associated with blood pressure, lowest platelet count, serum transaminases, serum creatinine, proteinuria, hematuria, pleural effusion, abdominal pain, persistent vomiting, rash, restlessness, serositis, and bleeding manifestations. Monitoring these parameters is useful for the effective management of dengue.

Keywords: Dengue, Platelet Count, Serositis, Severe Dengue
\end{abstract}

Citation: Ajmera RP, Kulkarni SV. A study of prognostic markers for dengue infection. Int JTravel Med Glob Health. 2021;9(3):139-145. doi:10.34172/ ijtmgh.2021.23.

\section{Introduction}

Dengue is an acute mosquito-borne viral disease and a global public health issue, extremely affecting the tropical and sub-tropical countries. ${ }^{1}$ Over $50-100$ million people are infected with dengue annually, while $>3.97$ billion people are at risk of contracting the disease. ${ }^{1,2}$ In the past five decades, the prevalence of dengue has exhibited a 30 -fold increase. ${ }^{1,2}$ Dengue is endemic in nearly 100 countries, including India. ${ }^{3}$ In India, annually, 390 million people are infected with dengue, and $>5.7$ million patients suffer from severe dengue. ${ }^{1}$

In 2009, the World Health Organization (WHO) dengue case classification was revised to classify dengue infection into two forms, namely dengue and severe dengue, which was introduced to simplify 1997 WHO dengue case classification comprising dengue fever (DF), dengue hemorrhagic fever (DHF), and dengue shock syndrome (DSS). ${ }^{4}$ Dengue is a complex, dynamic, and rapidly progressing disease, mediated by intricate interactions between the vector, virus, and host. ${ }^{3}$ Often, DF rapidly progresses to its severe form, i.e., DHF or DSS, which are the foremost causes of morbidity and mortality. ${ }^{5,6}$ Nevertheless, appropriate disease management effectively improves patient outcome. ${ }^{6}$

Considering the unavailability of antiviral drugs, disease management is primarily based on early supportive treatment. Thus, early diagnosis of patients at risk of developing a severe prognosis is crucial. ${ }^{7}$ Potential predictors of prognosis comprise clinical symptoms and laboratory parameters, including fever, body ache, skin rash, platelet/blood cell count, biomarkers, including liver enzymes, and markers of vascular functions. ${ }^{7}$ Various clinical, radiological, laboratory and immunological parameters predict the severity of dengue, permitting early disease management and reduction in morbidity and mortality. ${ }^{8}$

Limited studies have analyzed dengue in different demographic settings in the Indian subcontinent. ${ }^{1}$ Moreover, to our knowledge, only a few studies have investigated prognostic markers for dengue among the adult population of India. ${ }^{1,3}$ Such research should be conducted to better comprehend the burden of dengue and design appropriate therapeutic interventions for improved quality of life of

Copyright $(C 2021$ The Author(s). This is an open-access article distributed under the terms of the Creative Commons Attribution License (http:// creativecommons.org/licenses/by/4.0), which permits unrestricted use, distribution, and reproduction in any medium, provided the original work is properly cited. 
patients. ${ }^{1}$ The present study aimed to evaluate clinical, laboratory, and radiological factors predicting prognosis in dengue infection.

\section{Methods}

\section{Study Design and Sampling}

This prospective observational study enrolled 250 patients seropositive for dengue in Ramaiah Medical College, Bengaluru, Karnataka, India from October 2014 to June 2016. The study was approved by the institutional ethics committee, and written informed consent was obtained from all the study participants. Inclusion criteria included patients who were $>18$ years old and seropositive [nonstructural protein 1 (NS1) antigen-positive and/or IgM antibody positive] for dengue. Those who presented with mixed infections, such as dengue with malaria and those with any other systemic bleeding and/or coagulation disorders were excluded from the study.

\section{Sample Size}

A sample size of 250 was calculated as described by WHO using a random sampling method with absolute precision of $3 \%$ and desired confidence level of $95 \% .{ }^{10}$

\section{Data Collection}

Routine investigations, including complete blood test, platelet count, packed cell volume, liver function test with liver enzyme analysis, renal function test, and serum sodium level, along with routine urine analysis, including urine protein and red blood cell count, were performed in a diagnostic laboratory of the hospital under study. Moreover, clinical spectrum, abdominal ultrasonography, and chest X-ray were evaluated and correlated with the course of illness and with each other.

According to the National Guidelines for Clinical Management of Dengue Fever (2014), study patients were classified into three groups as per their symptoms, i.e., DF, DHF, and DSS. ${ }^{9}$ This classification was conducted at two-time points, upon admission and at the end of the hospital course. Considering that DF can progress to DHF or DSS throughout illness, patients were further analyzed by categorizing them into two groups. Those having DF both on admission and final diagnosis were classified under group $1(\mathrm{~N}=172 ; 89.1 \%)$, whereas those having DF on admission who later developed DHF or DSS were classified under group $2(\mathrm{~N}=21 ; 10.9 \%)$. Variables were compared between both groups, labeled as second analysis hereafter. A total of 193 patients were included in the second analysis, while 57 patients, i.e., 54 (21.6\%) patients with DHF and 3 (1.2\%) with DSS on admission, were excluded from the second analysis.

\section{Statistical Analysis}

Data were analyzed using statistical software R 3.6.1. All the parameters were not normally distributed and were accordingly analyzed. Qualitative parameters were assessed and expressed as percentages. Quantitative parameters were analyzed and expressed as mean \pm standard deviation. A univariate chi-square test was performed to investigate the association between the study parameters and the course of the disease. $P<0.05$ was considered statistically significant.

\section{Results}

Based on the status of dengue serology, 173 (69.2\%), 47 $(18.8 \%)$, and $30(12 \%)$ patients exhibited the presence of NS1 antigen, dengue-specific IgM antibodies, and both, respectively. Table 1 presents an assessment of dengue on admission and the final diagnosis.

On final diagnosis, the number of patients with DF had decreased by $8.4 \%$, whereas the number of patients with DHF and DSS had increased by $3.6 \%$ and $4.8 \%$, respectively.

Of 250 patients, 166 (66.4\%) were males and 84 (33.6\%) were females. Of 193 patients in the second analysis, in group $1,121(70.3 \%)$ were males and $51(29.7 \%)$ were females, and in group 2, 10 (47.6\%) were males and 11 (52.4\%) were females. The sex of patients was not associated with the final diagnosis $(P=0.088)$ and second analysis $(P=0.06)$. Age was also not associated with the final diagnosis $(P=0.57)$ and second analysis $(P=0.61)$ The patients were classified based on their age as depicted in Table 2.

Table 3 depicts the classification of patients based on quantitative parameters.

Final diagnosis correlated significantly with systolic blood pressure $(P<0.004)$, lowest platelet count $(P<0.001)$, and serum glutamic-oxaloacetic transaminase (SGOT) $(P=0.001)$. The second analysis correlated significantly with the lowest platelet count $(P=0.002)$ and SGOT $(P<0.001)$.

Table 4 depicts the classification of patients based on qualitative parameters.

Final diagnosis correlated significantly with urine protein $(P<0.001)$, urine red blood cells or RBCs $(P<0.001)$, pleural effusion $(P=0.0064)$, and serositis $(P<0.001)$. The second analysis also correlated significantly with urine protein $(P<0.001)$, urine RBC $(P<0.001)$, and serositis $(P<0.001)$.

Table 5 presents the classification of patients based on their clinical variables.

The final diagnosis correlated significantly with vomiting $(P<0.001)$, rash $(P<0.001)$, restlessness $(P<0.001)$, and bleeding manifestations $(P<0.001)$. The second analysis correlated significantly with vomiting $(P<0.001)$, abdominal pain $(P=0.002)$, rash $(P=0.012)$, restlessness $(P=0.003)$, and bleeding manifestations $(P<0.001)$.

Table 1. Assessment of Dengue on Admission and Final Diagnosis

\begin{tabular}{lccc}
\hline \multirow{2}{*}{ Diagnosis } & \multicolumn{2}{c}{ Number of Patients, $\mathbf{N}=\mathbf{2 5 0} ; \mathbf{n}(\%)$} & \multirow{2}{*}{$\%$ Change } \\
\cline { 2 - 3 } & On Admission & Final Diagnosis & \\
\hline DF & $193(77.2)$ & $172(68.8)$ & -8.4 \\
DHF & $54(21.6)$ & $63(25.2)$ & +3.6 \\
DSS & $3(1.2)$ & $15(6.0)$ & +4.8 \\
\hline
\end{tabular}

DF: dengue fever, DHF: dengue hemorrhagic fever, DSS: dengue shock syndrome. 
Table 2. Classification of Patients Based on Age

\begin{tabular}{|c|c|c|c|c|c|c|c|c|c|}
\hline \multirow[b]{2}{*}{ Variables } & \multicolumn{5}{|c|}{ Final Diagnosis } & \multicolumn{4}{|c|}{ Second Analysis } \\
\hline & $\begin{array}{c}D F, n=172 \\
n(\%)\end{array}$ & $\begin{array}{c}\text { DHF, } N=63 \\
n(\%)\end{array}$ & $\begin{array}{c}\text { DSS, } n=15 \\
n(\%)\end{array}$ & $\begin{array}{c}\text { Total, } \\
\mathrm{N}=250, \mathrm{n} \\
(\%)\end{array}$ & $P$ Value & $\begin{array}{c}\text { Group 1, } \\
n=172, n(\%)\end{array}$ & $\begin{array}{c}\text { Group 2, } \\
\mathrm{n}=21, \mathrm{n}(\%)\end{array}$ & $\begin{array}{c}\text { Total, } N=193 \\
n(\%)\end{array}$ & $P$ Value \\
\hline \multicolumn{10}{|l|}{ Gender } \\
\hline Male & $121(70.4)$ & $38(60.3)$ & $7(46.7)$ & $166(66.4)$ & \multirow{2}{*}{0.08} & $121(70.4)$ & $10(47.6)$ & $131(67.9)$ & \multirow{2}{*}{0.06} \\
\hline Female & $51(29.6)$ & $25(39.7)$ & $8(53.3)$ & $84(33.6)$ & & $51(29.6)$ & $11(52.4)$ & $62(32.1)$ & \\
\hline \multicolumn{10}{|l|}{ Age group } \\
\hline $18-30$ & $87(50.6)$ & $35(55.6)$ & $6(40.0)$ & $128(51.2)$ & \multirow{6}{*}{0.57} & $87(50.6)$ & $11(52.4)$ & $98(50.8)$ & \multirow{6}{*}{0.61} \\
\hline $31-40$ & $42(24.4)$ & $9(14.3)$ & $5(33.3)$ & $56(22.4)$ & & $42(24.4)$ & $4(19)$ & $46(23.8)$ & \\
\hline $41-50$ & $21(12.2)$ & $12(19)$ & $1(6.7)$ & $34(13.6)$ & & $21(12.2)$ & $5(23.8)$ & $26(13.5)$ & \\
\hline $51-60$ & $10(5.8)$ & $3(4.8)$ & $1(6.7)$ & $14(5.6)$ & & $10(5.8)$ & 0 & $14(7.3)$ & \\
\hline $61-70$ & $10(5.8)$ & $3(4.8)$ & $1(6.7)$ & $14(5.6)$ & & $10(5.8)$ & $1(4.8)$ & $11(5.7)$ & \\
\hline$>70$ & $2(1.2)$ & $1(1.6)$ & $1(6.7)$ & $4(1.6)$ & & $2(1.2)$ & 0 & $2(1)$ & \\
\hline
\end{tabular}

DF: dengue fever, DHF: dengue hemorrhagic fever, DSS: dengue shock syndrome; n (\%) represents number of patients.

\section{Discussion}

The prevalence of dengue is increasing globally, mainly in tropical and sub-tropical countries, due to climatic conditions, unclean environments especially in the rainy season, fast urbanization, and inefficiently planned urban colonization. ${ }^{1,11}$ Early diagnosis of patients and predicting prognosis of infection is crucial for better patient outcome. ${ }^{7}$ The study evaluated clinical, laboratory, and radiological factors predicting prognosis in dengue infection.

The present study included patients with NS1 antigen and IgM antibodies. NS1 antigen is one of the earliest markers identified in the blood of a patient with dengue within one to nine days of infection. ${ }^{12}$ IgM antibodies are the first antibodies to appear and are identifiable in nearly $50 \%$ of patients from the third or fifth day onward until the second or third month. ${ }^{6,12}$ The presence of NS1 and IgM antibodies indicates a recent/active infection. ${ }^{12}$

Male sex is commonly associated with the incidence of dengue. ${ }^{13}$ With respect to age, in the present study, dengue infection and the course of illness were reportedly independent of age. Literature suggests that young age is more commonly associated with the incidence of dengue. ${ }^{14}$ Contrastingly, literature also suggests that adults are more likely to contract dengue infection than children. ${ }^{15}$ In Southeast Asia, the incidence of DF and DHF is high among children, whereas, in Western countries, the incidence is high among adults. ${ }^{16}$ Reportedly, the association between dengue infection and age needs to be studied more extensively. ${ }^{15}$ Moreover, in a study conducted by Chau et al., regardless of immunological immaturity, <6-month-old infants infected with dengue presented vigorous neutralizing antibody responses that lasted $>1$ year after the infection. ${ }^{17}$ Thus, in line with the findings of Chau et al., in the present study, IgM antibodies and NS1 antigen could have attributed, to some extent, to the study findings in which the prognosis of dengue is independent of age. ${ }^{15}$ Moreover, in the present study, patients could not be equally distributed in the respective age groups, and over $50 \%$ of patients were in the age group of $18-30$ years, which also could have influenced the study findings.
Of all hematological predictors of dengue, the platelet count is the most common. As observed in the present study, platelet count decreases as the infection progresses. The lowest platelet count of 10-20 thousand/cumm is often observed in case of severe infection, which is an indicator for bleeding. ${ }^{2,13}$ However, the lowest platelet count alone cannot strongly predict the incidence of bleeding and is dependent on liver function status and coagulation parameters. ${ }^{2,13,18}$

In the present study, white blood cell or white blood cell (WBC) count was observably low on the final diagnosis, concurrent with the literature. ${ }^{18,19}$ Of note, WBC count is often consistently low throughout illness. ${ }^{19}$ This could be the reason that in the present study, the final diagnosis was independent of the WBC count. However, a normal WBC count was also observed in 89 (35.6\%) patients. Reportedly, WBC count can be within the normal range in severe dengue owing to physiological stress. ${ }^{18}$

Elevated levels of liver enzymes are often present in severe dengue. Concurrent with the literature, in the present study, SGOT and SGPT levels were remarkably high in DHF and DSS cases. ${ }^{18}$ Such high levels exhibit the severity of the hepatocellular injury. ${ }^{20}$ However, levels of liver enzymes peak typically around the second week of infection and thus should be carefully evaluated for prognosis. ${ }^{13}$

Increased serum creatinine levels and proteinuria observed in dengue commonly indicate kidney dysfunction. ${ }^{13,21}$ Degree of proteinuria indicates the severity of the infection and presents a manifestation of the pathogenic mechanisms triggered by the dengue virus on the lymphoreticular system, which subsequently leads to glomerular leakage, suggesting an incidence of DHF. ${ }^{22}$ Reportedly, urine protein/creatinine ratio is a potential predictor for $\mathrm{DF}$, where a higher ratio is more common in DHF than DSS. ${ }^{13}$ Plasma leakage, suggesting hypoalbuminemia due to dengue, is an indicator of severity. ${ }^{23}$ However, the severity of the infection cannot be well differentiated based on serum albumin levels. ${ }^{13}$ This may be due to the fact that in the present study, the final diagnosis was independent of serum albumin levels. Hyponatremia was also observed in $124(49.6 \%)$ patients. Although the final 
Table 3. Classification of Patients According to Quantitative Study Parameters

\begin{tabular}{|c|c|c|c|c|c|c|c|c|c|c|}
\hline \multirow[b]{2}{*}{ Parameter } & \multirow{2}{*}{$\begin{array}{c}\text { No. of } \\
\text { Patients, } \\
\mathrm{N}=250, \mathrm{n}(\%)\end{array}$} & \multicolumn{5}{|c|}{ Final Diagnosis } & \multicolumn{4}{|c|}{ Second Analysis } \\
\hline & & $\begin{array}{l}D F, n=172 \\
\text { mean } \pm S D\end{array}$ & $\begin{array}{c}\mathrm{DHF}, \mathrm{n}=63 \\
\text { mean } \pm \mathrm{SD}\end{array}$ & $\begin{array}{c}\mathrm{DSS}, \mathrm{n}=15 \\
\text { mean } \pm \mathrm{SD}\end{array}$ & $\begin{array}{c}\text { Total, } \mathrm{N}=\mathbf{2 5 9} \\
\text { mean } \pm \mathrm{SD}\end{array}$ & $P$ Value & $\begin{array}{c}\text { Group 1, } \\
n=172, \\
\text { mean } \pm S D\end{array}$ & $\begin{array}{c}\text { Group 2, } \\
\mathrm{n}=21, \text { mean } \\
\quad \pm \mathrm{SD}\end{array}$ & $\begin{array}{c}\text { Total, } \mathrm{N}=193 \\
\text { mean } \pm \mathrm{SD}\end{array}$ & $P$ Value \\
\hline \multicolumn{11}{|c|}{ Pulse rate (bpm) } \\
\hline$<60$ & $67(26.8)$ & \multirow{3}{*}{$\begin{array}{c}68.84 \pm \\
11.98\end{array}$} & \multirow{3}{*}{$68.21 \pm 12.14$} & \multirow{3}{*}{$63.20 \pm 11.90$} & \multirow{3}{*}{$68.34 \pm 12.04$} & \multirow{3}{*}{0.156} & \multirow{3}{*}{$68.84 \pm 11.98$} & \multirow{3}{*}{$67.14 \pm 15.09$} & \multirow{3}{*}{$68.65 \pm 12.32$} & \multirow{3}{*}{0.286} \\
\hline $60-90$ & $177(70.8)$ & & & & & & & & & \\
\hline$>90$ & $6(2.4)$ & & & & & & & & & \\
\hline \multicolumn{11}{|c|}{ Systolic blood pressure $(\mathrm{mm} \mathrm{Hg})$} \\
\hline$<100$ & $9(3.6)$ & \multirow{3}{*}{$\begin{array}{c}114.23 \pm \\
9.00\end{array}$} & \multirow{3}{*}{$113.71 \pm 9.11$} & \multirow{3}{*}{$\begin{array}{c}103.60 \pm \\
15.03\end{array}$} & \multirow{3}{*}{$113.46 \pm 9.76$} & \multirow{3}{*}{$<0.004^{*}$} & & & & \\
\hline $100-120$ & $215(86.0)$ & & & & & & $\begin{array}{c}114.23 \pm \\
9.00\end{array}$ & $\begin{array}{c}110.10 \pm \\
10.32\end{array}$ & $113.78 \pm 9.22$ & 0.085 \\
\hline$>120$ & $26(10.4)$ & & & & & & & & & \\
\hline Diastolic blo & ressure $(\mathrm{mm} \mathrm{Hg})$ & & & & & & & & & \\
\hline $60-90$ & $154(61.6)$ & $73.76 \pm 5.84$ & $72.38 \pm 6.52$ & $69.87 \pm 10.65$ & $73.18 \pm 6.44$ & 0.109 & $73.76 \pm 5.84$ & $71.52 \pm 8.07$ & $73.51 \pm 6.14$ & 0.121 \\
\hline$>90$ & $95(38.0)$ & & & & & & & & & \\
\hline HCT levels $\left(^{\circ}\right.$ & & & & & & & & & & \\
\hline$<40$ & $89(35.6)$ & & & & & & & & & \\
\hline $40.1-49.9$ & $156(62.4)$ & $41.22 \pm 4.09$ & $39.91 \pm 5.07$ & $39.27 \pm 5.48$ & $39.27 \pm 5.48$ & 0.156 & $41.22 \pm 4.09$ & $39.51 \pm 5.01$ & $41.03 \pm 4.22$ & 0.179 \\
\hline$>50$ & $5(2.0)$ & & & & & & & & & \\
\hline WBC count & sand/cumm) & & & & & & & & & \\
\hline$<4000$ & $155(62.0)$ & & & & & & & & & \\
\hline 4000-10000 & 89 (35.6) & $\begin{array}{c}4180.41 \pm \\
2083.7\end{array}$ & $\begin{array}{c}4115.90 \pm \\
2609.12\end{array}$ & $\begin{array}{c}5035.33 \pm \\
2413.02\end{array}$ & $\begin{array}{c}4215.45 \pm \\
2246.78\end{array}$ & 0.087 & $\begin{array}{l}4180.41 \pm \\
2083.7\end{array}$ & $\begin{array}{c}4925.24 \pm \\
2802.12\end{array}$ & $\begin{array}{c}4261.45 \pm \\
2176.9\end{array}$ & 0.245 \\
\hline$>10000$ & $6(2.4)$ & & & & & & & & & \\
\hline Platelet coun & usand/cumm) & & & & & & & & & \\
\hline$<20$ & $31(12.4)$ & & & & & & & & & \\
\hline $20-50$ & $68(27.2)$ & $\begin{array}{c}57.55 \pm \\
30.12\end{array}$ & $52.92 \pm 30.80$ & $52.00 \pm 45.92$ & $56.05 \pm 31.35$ & 0.279 & $57.55 \pm 30.12$ & $50.24 \pm 32.42$ & $56.76 \pm 30.37$ & 0.186 \\
\hline$>50$ & $151(60.4)$ & & & & & & & & & \\
\hline Lowest platel & & & & & & & & & & \\
\hline$<10$ & $6(2.4)$ & & & & & & & & & \\
\hline $10-20$ & $103(41.2)$ & $\begin{array}{c}27.74 \pm \\
14.13\end{array}$ & $16.19 \pm 4.98$ & $31.60 \pm 25.09$ & $25.06 \pm 14.36$ & $<0.001 *$ & $\begin{array}{c}27.74 \pm \\
14.13\end{array}$ & $18.00 \pm 8.12$ & $26.68 \pm 13.93$ & $<0.001 *$ \\
\hline$>20$ & $141(56.4)$ & & & & & & & & & \\
\hline SGOT level ( & & & & & & & & & & \\
\hline$<100$ & $106(42.4)$ & & & & & & & & & \\
\hline $101-200$ & $101(40.4)$ & $\begin{array}{c}130.52 \pm \\
117.06\end{array}$ & $\begin{array}{c}230.95 \pm \\
604.38\end{array}$ & $\begin{array}{c}371.07 \pm \\
476.58\end{array}$ & $\begin{array}{c}170.26 \pm \\
342.90\end{array}$ & $0.001 *$ & $\begin{array}{c}130.52 \pm \\
117.06\end{array}$ & $\begin{array}{c}412.52 \pm \\
1035.64\end{array}$ & $\begin{array}{l}161.2 \pm \\
362.88\end{array}$ & $0.011^{*}$ \\
\hline$>200$ & $43(17.2)$ & & & & & & & & & \\
\hline SGPT level (L & & & & & & & & & & \\
\hline$<100$ & $108(43.2)$ & & & & & & & & & \\
\hline $101-200$ & $105(42.0)$ & $\begin{array}{c}133.92 \pm \\
106.11\end{array}$ & $\begin{array}{c}142.40 \pm \\
208.01\end{array}$ & $\begin{array}{c}220.33 \pm \\
227.36\end{array}$ & $\begin{array}{c}141.24 \pm \\
147.74\end{array}$ & 0.621 & $\begin{array}{c}133.92 \pm \\
106.11\end{array}$ & $\begin{array}{c}212.38 \pm \\
351.92\end{array}$ & $\begin{array}{c}142.46 \pm \\
153.39\end{array}$ & 0.526 \\
\hline$>200$ & $37(14.8)$ & & & & & & & & & \\
\hline Serum album & vel $(g / d L)$ & & & & & & & & & \\
\hline$<3.5$ & $202(80.8)$ & 3 & 32 & 1 & & 0671 & & & & \\
\hline$>3.5$ & $48(19.2)$ & $-3.35 \pm 0.35$ & $3.33 \pm 0.32$ & $3.18 \pm 0.68$ & $3.34 \pm 0.37$ & 0.671 & $3.35 \pm 0.35$ & $3.42 \pm 0.42$ & $3.36 \pm 0.36$ & 0.267 \\
\hline Serum creati & level (mg/dL) & & & & & & & & & \\
\hline$<1.2$ & $234(93.6)$ & & & & & & & & & \\
\hline$>1.2$ & $16(6.4)$ & $-0.82 \pm 0.21$ & $0.77 \pm 0.21$ & $0.97 \pm 0.46$ & $0.82 \pm 0.24$ & 0.154 & $0.82 \pm 0.21$ & $0.73 \pm 0.21$ & $0.81 \pm 0.21$ & 0.071 \\
\hline Serum sodiur & el $(\mathrm{mEq} / \mathrm{L})$ & & & & & & & & & \\
\hline$<135$ & $124(49.6)$ & $134.49 \pm$ & 87 & 87 & $2+280$ & 0670 & $134.49 \pm$ & 1227 & 50 & $0 ?-0$ \\
\hline
\end{tabular}

DF: dengue fever, DHF: dengue hemorrhagic fever, DSS: dengue shock syndrome; HCT: Hematocrit; WBC: White blood cells; SGOT: Serum glutamicoxaloacetic transaminase; SGPT: Serum glutamic pyruvic transaminase. 
Table 4. Classification of Patients According to Qualitative Study Parameters

\begin{tabular}{|c|c|c|c|c|c|c|c|c|c|}
\hline \multirow[b]{2}{*}{ Parameter } & \multicolumn{5}{|c|}{ Final diagnosis } & \multicolumn{4}{|c|}{ Second analysis } \\
\hline & $\begin{array}{c}\text { DF, } \\
\mathrm{N}=172, \mathrm{n} \\
(\%)\end{array}$ & $\begin{array}{l}\text { DHF, } \\
N=63, \\
\text { n (\%) }\end{array}$ & $\begin{array}{l}\text { DSS, } \\
N=15, \\
\text { n (\%) }\end{array}$ & $\begin{array}{c}\text { Total, } \\
\mathrm{N}=\mathbf{2 5 0}, \mathrm{n} \\
(\%)\end{array}$ & $P$ value & $\begin{array}{c}\text { Group 1, } \\
\begin{array}{c}\mathrm{N}=172, \\
\mathrm{n}(\%)\end{array}\end{array}$ & $\begin{array}{c}\text { Group 2, } \\
\text { N=21, } \\
\text { n (\%) }\end{array}$ & $\begin{array}{c}\text { Total, } \\
\mathrm{N}=193 \\
\text { n (\%) }\end{array}$ & $P$ value \\
\hline \multicolumn{10}{|c|}{ Urine Protein (Per High Power Field) } \\
\hline Absent & $138(80.2)$ & $15(23.8)$ & $2(13.3)$ & $155(62.0)$ & \multirow{2}{*}{$<0.001^{*}$} & $138(80.2)$ & $8(38.1)$ & $146(75.6)$ & \multirow{2}{*}{$<0.001^{*}$} \\
\hline Present & $34(19.8)$ & $48(76.2)$ & $13(86.7)$ & $95(38.0)$ & & $34(19.8)$ & $13(61.9)$ & $47(24.4)$ & \\
\hline \multicolumn{10}{|c|}{ Urine RBC (Per High Power Field) } \\
\hline Absent & $150(87.2)$ & $37(58.7)$ & $3(20)$ & $190(76.0)$ & \multirow{2}{*}{$<0.001^{*}$} & $150(87.2)$ & $11(52.38)$ & $161(83.42)$ & \multirow{2}{*}{$<0.001^{*}$} \\
\hline Present & $22(12.8)$ & $26(41.3)$ & $12(80)$ & $60(24.0)$ & & $22(12.8)$ & $10(47.62)$ & $32(16.58)$ & \\
\hline \multicolumn{10}{|c|}{ Pleural Effusion on Chest X-Ray } \\
\hline Absent & $126(73.26)$ & $35(55.56)$ & $6(40)$ & $167(66.8)$ & \multirow{2}{*}{$0.0064^{*}$} & $126(73.26)$ & $16(76.20)$ & $142(73.58)$ & \multirow{2}{*}{0.793} \\
\hline Present & $46(26.74)$ & $28(44.44)$ & $9(60)$ & $83(33.2)$ & & $46(26.74)$ & $5(23.80)$ & $51(26.42)$ & \\
\hline \multicolumn{10}{|c|}{ Serositis } \\
\hline Absent & 87 (50.6) & $1(1.59)$ & 0 & 88 (35.2) & & 87 (50.6) & 0 & 87 (45.1) & \multirow{4}{*}{$<0.001^{*}$} \\
\hline Ascites only & $38(22.1)$ & $20(31.7)$ & $4(26.7)$ & $62(24.8)$ & & $38(22.1)$ & $6(28.6)$ & $44(22.8)$ & \\
\hline $\begin{array}{l}\text { Pleural effusion } \\
\text { only }\end{array}$ & $21(12.2)$ & $23(36.5)$ & $3(20)$ & 47 (18.8) & $<0.001^{*}$ & $21(12.2)$ & $12(57.1)$ & $33(17.1)$ & \\
\hline $\begin{array}{l}\text { Both ascites and } \\
\text { pleural effusion }\end{array}$ & $26(15.1)$ & $19(30.2)$ & $8(53.3)$ & $53(21.2)$ & & $26(15.1)$ & $3(14.3)$ & $29(15)$ & \\
\hline
\end{tabular}

DF: dengue fever, DHF: dengue hemorrhagic fever, DSS: dengue shock syndrome; RBC: Red blood cells; $\mathrm{n}(\%)$ represents number of patients, Chi square test.

Table 5. Classification of Patients According to Clinical Variables

\begin{tabular}{|c|c|c|c|c|c|c|c|c|c|c|}
\hline \multirow[b]{2}{*}{ Clinical variables } & & \multicolumn{5}{|c|}{ Final diagnosis } & \multicolumn{4}{|c|}{ Second analysis } \\
\hline & & $\begin{array}{c}\text { DF, } n=172 \\
n(\%)\end{array}$ & $\begin{array}{c}\text { DHF, } n=63 \\
\text { n (\%) }\end{array}$ & $\begin{array}{c}\text { DSS, } n=15 \\
n(\%)\end{array}$ & $\begin{array}{c}\text { Total, } \\
\mathrm{N}=\mathbf{2 5 0 , \mathrm { n }} \\
(\%)\end{array}$ & $P$ value & $\begin{array}{c}\text { Group 1, } \\
\mathrm{n}=172, \mathrm{n} \\
(\%)\end{array}$ & $\begin{array}{c}\text { Group 2, } \\
\mathrm{n}=21, \mathrm{n}(\%)\end{array}$ & $\begin{array}{c}\text { Total, } N=193 \\
\text { n }(\%)\end{array}$ & $P$ Value \\
\hline \multirow[t]{2}{*}{ Fever } & Yes & $163(94.8)$ & 62 (98.4) & $15(100)$ & $240(96)$ & \multirow{2}{*}{0.323} & $163(94.8)$ & $20(95.2)$ & $183(94.8)$ & \multirow{2}{*}{0.999} \\
\hline & No & $9(5.23)$ & $1(1.59)$ & 0 & $10(4)$ & & $9(5.2)$ & $1(4.8)$ & $10(5.2)$ & \\
\hline \multirow[t]{2}{*}{ Vomiting } & Yes & $71(41.3)$ & $48(76.2)$ & $15(100)$ & $134(53.6)$ & \multirow{2}{*}{$<0.0001^{*}$} & $71(41.3)$ & $18(85.7)$ & $89(46.1)$ & \multirow{2}{*}{$<0.0001^{*}$} \\
\hline & No & $101(58.72)$ & $15(23.81)$ & 0 & $116(46.4)$ & & $101(58.7)$ & $3(14.3)$ & $104(53.9)$ & \\
\hline \multirow[t]{2}{*}{ Abdominal pain } & Yes & $19(11)$ & $11(17.5)$ & $3(20)$ & $33(13.2)$ & \multirow{2}{*}{0.317} & $19(11.1)$ & $8(38.1)$ & $27(14)$ & \multirow{2}{*}{$0.002 *$} \\
\hline & No & 153 (88.95) & $52(82.54)$ & $12(80)$ & 217 (86.8) & & 153 (88.9) & $13(61.9)$ & $166(86)$ & \\
\hline \multirow[t]{2}{*}{ Headache } & Yes & $146(84.9)$ & $54(85.7)$ & $10(66.7)$ & $210(84)$ & \multirow{2}{*}{0.166} & $146(84.9)$ & $20(95.2)$ & $166(86)$ & \multirow{2}{*}{0.338} \\
\hline & No & $26(15.12)$ & $9(14.29)$ & $5(33.33)$ & $40(16)$ & & $26(15.1)$ & $1(4.8)$ & $27(14)$ & \\
\hline \multirow[t]{2}{*}{ Body ache } & Yes & $152(88.4)$ & $58(92.1)$ & $13(86.7)$ & $223(89.2)$ & \multirow{2}{*}{0.684} & $152(88.4)$ & 19 (90.5) & $171(88.9)$ & \multirow{2}{*}{0.999} \\
\hline & No & $20(11.63)$ & $5(7.94)$ & $2(13.33)$ & $27(10.8)$ & & $20(11.6)$ & $2(9.5)$ & $22(11.4)$ & \\
\hline \multirow[t]{2}{*}{ Retro-orbital pain } & Yes & $6(3.5)$ & $5(7.9)$ & $2(13.3)$ & $13(5.2)$ & \multirow{2}{*}{0.135} & $6(3.5)$ & $2(9.5)$ & $8(4.1)$ & \multirow{2}{*}{0.465} \\
\hline & No & $166(96.51)$ & $58(92.06)$ & 13 (86.67) & 237 (94.8) & & $166(96.5)$ & 19 (90.5) & 185 (95.9) & \\
\hline \multirow[t]{2}{*}{ Rash (maculopapular) } & Yes & $19(11)$ & 25 (39.7) & $5(33.3)$ & 49 (19.6) & \multirow{2}{*}{$<0.0001^{*}$} & $19(11)$ & $7(33.3)$ & $26(13.5)$ & \multirow{2}{*}{$0.012^{*}$} \\
\hline & No & $153(88.95)$ & $38(60.32)$ & $10(66.67)$ & $201(80.4)$ & & $153(89)$ & $14(66.7)$ & $167(86.5)$ & \\
\hline \multirow[t]{2}{*}{ Restlessness } & Yes & 0 & $3(4.8)$ & $7(46.7)$ & $10(4)$ & \multirow{2}{*}{$<0.0001^{*}$} & 0 & $2(9.5)$ & $2(1)$ & \multirow{2}{*}{$0.003^{*}$} \\
\hline & No & $172(100)$ & $60(95.24)$ & $8(53.33)$ & $240(96)$ & & $172(100)$ & 19 (90.5) & 191 (99) & \\
\hline \multirow[t]{2}{*}{ Bleeding manifestations } & Yes & 0 & $63(100)$ & $15(100)$ & $79(31.5)$ & \multirow{2}{*}{$<0.0001^{*}$} & 0 & $10(47.6)$ & $10(5.2)$ & \multirow{2}{*}{$<0.000$} \\
\hline & No & $172(100)$ & 0 & 0 & $172(68.5)$ & & $172(100)$ & $11(52.4)$ & $183(94.8)$ & \\
\hline
\end{tabular}

DF: dengue fever, DHF: dengue hemorrhagic fever, DSS: dengue shock syndrome; $\mathrm{n}(\%)$ represents number of patients, chi square test. 
diagnosis was independent of serum sodium, presumably, the reduced levels of serum sodium present a higher incidence of DF-associated complications. ${ }^{24}$

Reportedly, a combination ofleucopenia, thrombocytopenia, elevated liver enzymes, and low C-reactive protein strongly predicts prognosis during the early phases of dengue. ${ }^{17,25}$ On admission, patients with dengue exhibit fever, vomiting, rash, aches or pain, bleeding manifestations, and clinical fluid accumulation, such as ascites or pleural effusion. ${ }^{14,26,27}$ Similar findings were noted in the present study. The presence of bleeding manifestations, pleural effusion, and ascites are strongly predictive of DSS. ${ }^{13,14}$

Skin involvement is prevalent in dengue. ${ }^{28}$ Owing to capillary permeability, there is an increase in leakage of plasma, RBCs, and electrolytes in patients with DHF and/or DSS. ${ }^{14}$ Skin rashes either dissolve entirely or generalize with progressing infection. ${ }^{28}$ In the present study, rashes persisted over the course of infection. In DHF, body temperature surpasses $39^{\circ} \mathrm{C}$ and persists for two to seven days. ${ }^{29}$ For those who develop DSS, health further deteriorates after prolonged fever. The clinical deterioration occurs as the temperature falls. ${ }^{29}$ Considering the course of high temperature, and as observed in the present study, fever cannot be considered as a prognostic marker for dengue. ${ }^{29}$ In severe cases, dengue damages the heart, lungs, or liver, and the blood pressure decreases remarkably, leading to shock or death. ${ }^{30}$

The study possesses certain limitations. Because only adult patients were enrolled, the findings cannot be extrapolated to pediatric patients, which is important considering the high prevalence of dengue among children. Patients did not receive post-discharge follow-up. Future studies with longterm follow-up would help understand the comprehensive prognosis of the infection. The study lacked data on the onset of the infection and did not evaluate immunological parameters as potential biomarkers. Although these might not have altered the study outcomes, they would have allowed a better comprehension of the prognosis of dengue.

The present study was conducted in a tertiary care hospital in Bengaluru, Karnataka. The findings may not apply to all parts of India, because although dengue is endemic in the Indian subcontinent, its clinical manifestations can be substantially altered based on epidemiological and climatic parameters, including temperature and rainfall. ${ }^{3}$ Thus, to validate the prognosis of dengue in India, future studies must span various states across the country and assess the influence of climatic parameters on the prognosis of dengue. The present study also provides a basis for establishing a prognostic prediction paradigm comprising clinical, radiological, and laboratory factors for dengue to understand the severity of the infection. The findings proffer sizeable data for appropriate triage and treatment. The findings also help identify high-risk patients and subsequently decrease the morbidity rates. Future studies with a gender-based classification of patients can be conducted to better understand the prognosis of the infection and implement appropriate effective treatment. Moreover, large-scale multi-centered trials are needed to confirm the present findings.

\section{Research Highlights}

\section{What Is Already Known?}

Dengue is a complex disease regulated by the complicated host-virus interactions. However, various clinical, radiological, laboratory and immunological parameters predict the severity of dengue, permitting early disease management and reduction in morbidity and mortality. Fascinatingly, the studies that have analyzed dengue in different demographic settings in the Indian subcontinent are rather limited. In addition to this, only a few studies have investigated prognostic markers for dengue in the adult population in India.

\section{What Does This Study Add?}

his study adds to the knowledge of predicting prognosis in dengue infection considering the various factors, including clinical, laboratory, and radiological; which would significantly contribute to early diagnosis and adequate management of dengue infection.

\section{Conclusion}

Blood pressure, lowest platelet count, SGOT, SGPT, serum creatinine, proteinuria, hematuria, pleural effusion, abdominal pain, persistent vomiting, bleeding manifestations, rash, restlessness, and serositis are predictive of severe dengue. These parameters should be implemented as prognostic markers in clinical practice for vigilant monitoring of the progress of dengue. Early anticipation of severe dengue allows the implementation of appropriate therapeutic interventions and can substantially decrease morbidity and mortality. These parameters can be implemented to design a scoring system that would predict the severity of dengue early in the course of the infection, and manage the same effectively.

\section{Authors' Contributions}

RPK developed the study concept and also contributed to the study design. Data collection and analysis were performed by both authors. Data were interpreted by SVK. Both authors approved the final version of the manuscript for submission.

\section{Conflict of Interest Disclosures}

The authors report no known conflict of interest.

\section{Ethical Approval}

This study was approved by the MS Ramaiah Medical College, Mathikere, Bangalore, Karnataka, and informed consent was taken from all participants.

\section{Funding/Support}

This research did not receive any specific grant from funding agencies in the public, commercial, or not-for-profit sectors.

\section{TReferences}

1. Shah PS, Alagarasu K, Karad S, et al. Seroprevalence and incidence of primary dengue infections among children in a rural region of Maharashtra, Western India. BMC Infect Dis. 2019;19(1):296. doi:10.1186/s12879-019-3937-z. 
2. Lee IK, Huang $\mathrm{CH}$, Huang WC, et al. Prognostic factors in adult patients with dengue: developing risk scoring models and emphasizing factors associated with death $\leq 7$ days after illness onset and $\leq 3$ days after presentation. J Clin Med. 2018;7(11):396. doi:10.3390/jcm7110396.

3. Mutheneni SR, Morse AP, Caminade C, Upadhyayula SM. Dengue burden in India: recent trends and importance of climatic parameters. Emerg Microbes Infect. 2017;6(8):e70. doi:10.1038/emi.2017.57.

4. Horstick O, Martinez E, Guzman MG, Martin JL, Ranzinger SR. WHO dengue case classification 2009 and its usefulness in practice: an expert consensus in the Americas. Pathog Glob Health. 2015;109(1):19-25. doi:10.1179/204777321 5y.0000000003.

5. da Silva MM, Gil LH, de Azevedo Marques ET Jr, CalzavaraSilva CE. Potential biomarkers for the clinical prognosis of severe dengue. Mem Inst Oswaldo Cruz. 2013;108(6):755-762. doi:10.1590/0074-0276108062013012.

6. Alejandria MM. Dengue haemorrhagic fever or dengue shock syndrome in children. BMJ Clin Evid. 2015;2015:0917.

7. Dao Phuoc T, Khuong Quynh L, Vien Dang Khanh L, et al. Clinical prognostic models for severe dengue: a systematic review protocol. Wellcome Open Res. 2019;4:12. doi:10.12688/ wellcomeopenres.15033.2.

8. Pongpan S, Wisitwong A, Tawichasri C, Patumanond J. Prognostic indicators for dengue infection severity. Int J Clin Pediatr. 2013;2(1):12-18. doi:10.4021/ijcp73w.

9. Government of India. National Guidelines for Clinical Management of Dengue Fever. [Cited on 01 July 2020]. Available from: http://www.mdumcmed.com/wp-content/ uploads/2016/02/Dengue-National-Guidelines-2014Compressed.pdf.

10. World Health Organization (WHO). Dengue: Guidelines for Diagnosis, Treatment, Prevention and Control. Geneva: WHO; 2009.

11. World Health Organization. Dengue increase likely during rainy season: WHO warns. [Cited on 07 July 2020]. Available from: https://www.who.int/westernpacific/news/detail/11-062019-dengue-increase-likely-during-rainy-season-who-warns.

12. Joshi AA, Divyashree BN, Gayathri BR. Hematological parameters in dengue: the serological angle a study. Int J Hematol Res. 2018;4(1):180-184. doi:10.17554/j.issn.24093548.2018.04.49.

13. Yacoub S, Wills B. Predicting outcome from dengue. BMC Med. 2014;12:147. doi:10.1186/s12916-014-0147-9.

14. Chacko B, Subramanian G. Clinical, laboratory and radiological parameters in children with dengue fever and predictive factors for dengue shock syndrome. J Trop Pediatr. 2008;54(2):137140. doi:10.1093/tropej/fmm084.

15. Egger JR, Coleman PG. Age and clinical dengue illness. Emerg Infect Dis. 2007;13(6):924-925. doi:10.3201/eid1306.070008.

16. Cavalcanti LP, Vilar D, Souza-Santos R, Teixeira MG. Change in age pattern of persons with dengue, northeastern Brazil. Emerg
Infect Dis. 2011;17(1):132-134. doi:10.3201/eid1701.100321.

17. Chau TN, Hieu NT, Anders KL, et al. Dengue virus infections and maternal antibody decay in a prospective birth cohort study of Vietnamese infants. J Infect Dis. 2009;200(12):18931900. doi:10.1086/648407.

18. Prabhavathi R, Madhusudan SR, Suman MG, Govindaraj M, Puttaswamy M. Study of clinical and laboratory predictive markers of dengue fever and severe dengue in children. Int J Pediatr Res. 2017;4(6):397-404. doi:10.17511/ijpr.2017.i06.08.

19. Jain H. Clinical profile and outcome of dengue fever in hospitalized children of South Rajasthan, India. Int J Contemp Pediatr. 2016 Apr;3(2):546-549. doi:10.18203/2349-3291. ijcp20161035.

20. Azin FR, Gonçalves RP, Pitombeira MH, Lima DM, Branco IC. Dengue: profile of hematological and biochemical dynamics. Rev Bras Hematol Hemoter. 2012;34(1):36-41. doi:10.5581/1516-8484.20120012.

21. Phakhounthong K, Chaovalit P, Jittamala P, et al. Predicting the severity of dengue fever in children on admission based on clinical features and laboratory indicators: application of classification tree analysis. BMC Pediatr. 2018;18(1):109. doi:10.1186/s12887-018-1078-y.

22. Vasanwala FF, Thein TL, Leo YS, et al. Predictive value of proteinuria in adult dengue severity. PLoS Negl Trop Dis. 2014;8(2):e2712. doi:10.1371/journal.pntd.0002712.

23. Poornima Shankar PB. Biochemical parameters (lactate dehydrogenase, serum albumin) as early predictor of severe dengue. Int J Contemp Pediatr. 2017;4(2):464-469. doi:10.18203/2349-3291.ijcp20170690.

24. Reddy AA, Reddy TP, Pranam GM, Pranam U, Manjunathe GA. Serum sodium as a prognostic marker in dengue fever cases admitted to PICU in Navodaya hospital, Raichur, India. Int J Contemp Pediatr. 2017;4(1):222-225. doi:10.18203/23493291.ijcp20164608.

25. Ho TS, Wang SM, Lin YS, Liu CC. Clinical and laboratory predictive markers for acute dengue infection. J Biomed Sci. 2013;20(1):75. doi:10.1186/1423-0127-20-75.

26. Centers for Disease Control and Prevention. Dengue. [Cited on 01 July 2020]. Available from: https://www.cdc.gov/dengue/ symptoms/index.html.

27. Schaefer TJ, Panda PK, Wolford RW. Dengue fever. In: StatPearls. Treasure Island, FL: StatPearls Publishing; 2019.

28. Mishra AK, George AA, Abhilash KPP. The relationship between skin rash and outcome in dengue. J Vector Borne Dis. 2018;55(4):310-314. doi:10.4103/0972-9062.256567.

29. World Health Organization. Clinical Diagnosis. [Cited on 01 July 2020]. Available from: https://www.who.int/csr/resources/ publications/dengue/012-23.pdf?ua $=1$.

30. Mayo clinic. Dengue fever. [Cited on 01 July 2020]. Available from: https://www.mayoclinic.org/diseases-conditions/ dengue-fever/symptoms-causes/syc-20353078\#: :text = If\%20 severe $\% 2$ C $\% 20$ dengue $\% 20$ fever $\% 20$ can, $\% 2$ C $\% 20$ in $\% 20$ some $\% 20$ cases $\% 2 \mathrm{C} \% 20$ death. 